\title{
Combustion Characteristics of Nanoaluminium-Based Composite Solid Propellants: An Overview
}

\author{
Kandasamy Jayaraman, ${ }^{1}$ Ponnurengam Malliappan Sivakumar ${ }^{(D)}{ }^{2}$ Ali Zarrabi $\mathbb{D}^{3}{ }^{3}$ \\ R. Sivakumar $\mathbb{D}^{4}$ and S. Jeyakumar $\mathbb{D}^{5}$ \\ ${ }^{1}$ Department of Mechanical Engineering, Middle East Technological University, Ankara, Turkey \\ ${ }^{2}$ Center for Molecular Biology, Institute of Research and Development, Duy Tan University, 03 Quang Trung, Da Nang, Vietnam \\ ${ }^{3}$ Sabanci University Nanotechnology Research and Application Center (SUNUM), Tuzla, Istanbul 34956, Turkey \\ ${ }^{4}$ Department of Mechanical Engineering, Vellore Institute of Technology, Chennai, India \\ ${ }^{5}$ Department of Mechanical Engineering, Kalasalingam Academy of Research and Education, Krishnankoil, India
}

Correspondence should be addressed to Kandasamy Jayaraman; jayaraman78@gmail.com

Kandasamy Jayaraman and Ponnurengam Malliappan Sivakumar contributed equally to this work.

Received 4 February 2021; Accepted 2 May 2021; Published 19 May 2021

Academic Editor: Muhammad J. Habib

Copyright (c) 2021 Kandasamy Jayaraman et al. This is an open access article distributed under the Creative Commons Attribution License, which permits unrestricted use, distribution, and reproduction in any medium, provided the original work is properly cited.

\begin{abstract}
The nanosized powders have gained attention to produce materials exhibiting novel properties and for developing advanced technologies as well. Nanosized materials exhibit substantially favourable qualities such as improved catalytic activity, augmentation in reactivity, and reduction in melting temperature. Several researchers have pointed out the influence of ultrafine aluminium $(\sim 100 \mathrm{~nm})$ and nanoaluminium $(<100 \mathrm{~nm})$ on burning rates of the composite solid propellants comprising AP as the oxidizer. The inclusion of ultrafine aluminium augments the burning rate of the composite propellants by means of aluminium particle's ignition through the leading edge flames (LEFs) anchoring above the interfaces of coarse AP/binder and the binder/fine AP matrix flames as well. The sandwiches containing $15 \%$ of nanoaluminium solid loading in the binder lamina exhibit the burning rate increment of about $20-30 \%$. It was noticed that the burning rate increment with nanoaluminium is around 1.6-2 times with respect to the propellant compositions without aluminium for various pressure ranges and also for different micronsized aluminium particles in the composition. The addition of nano- $\mathrm{Al}$ in the composite propellants washes out the plateaus in burning rate trends that are perceived from non-Al and microaluminized propellants; however, the burning rates of nanoaluminized propellants demonstrate low-pressure exponents at the higher pressure level. The contribution of catalysts towards the burning rate in the nanoaluminized propellants is reduced and is apparent only with nanosized catalysts. The near-surface nanoaluminium ignition and diffusion-limited nano-Al particle combustion contribute heat to the propellant-regressing surface that dominates the burning rate. Quench-collected nanoaluminized propellant residues display notable agglomeration, although a minor percentage of the agglomerates are in the 1-3 $\mu \mathrm{m}$ range; however, these are within $5 \mu \mathrm{m}$ in size. Percentage of elongation and initial modulus of the propellant are decreased when the coarse AP particles are replaced by aluminium in the propellant composition.
\end{abstract}

\section{Introduction}

Solid propellants have been utilized in various applications such as space launch vehicles, missiles, and spacecrafts. Burning rate determination and control are the essential parameters during composite propellant development. A slight variation in the propellant ingredients' content may lead to big impact in the burning rates. Theoretical models have been developed to study the effect of flame structures, such as the granular diffusion flame (GDF) model [1] and the Beckstead-Derr-Price (BDP) model [2]. A lot of experiments have been carried out in the past to find out the effect of 
single/combined formulation parametric variations in the burning rates. The accomplishment of the "ultrafine" aluminium particle with a size of $\sim 100 \mathrm{~nm}$ or above in composite propellant combustion features has been reported by several investigators. The contribution of aluminium towards catalytic effects on the deflagration of ammonium perchlorate (AP) pellets was investigated by Romonadova and Pokhil [3], and burning rates of the dry-pressed mixtures of AP and ultrafine aluminium were also reported. Recently, several advancing research studies are carried out towards the production of nano-/ultrafine aluminium through various techniques and processes, and its performance is estimated in the solid propellant rockets, explosives, thermites, guns, etc. The propellant burning rates with the maximum pressure of 6.9 MPa were examined by Dokhan et al. [4] by utilising either unimodal ultrafine particles or micron-sized aluminium ones and also using bimodal mixtures of both sized particles. It was mentioned that the ultrafine aluminium addition enhances the burning rate of the propellant by means of near-surface aluminium particle ignition as supported by leading edge flames (LEFs) formed above the binder/coarse AP boundaries and also from the binder/fine AP matrix flames. Burning rate augmentation owing to nanoaluminium addition is about 1.6-2 times with respect to other nonaluminized and aluminized propellant counterparts for various pressures and aluminium contents, investigated by Ivanov et al. [5]. De Luca et al. [6] and Galfetti et al. $[7,8]$ reported the propellant burning rates that comprises of ultra-fine aluminium and also compared with nonaluminized propellant compositions which was tested up to the maximum pressure of around $7 \mathrm{MPa}$. And it was also noticed that burning rates almost increased twice due to the inclusion of ultrafine aluminium over the tested pressure ranges, i.e., it implies that the burning rate pressure exponent is not varied over the tested pressure ranges. Additionally, it was also stated that bimodal aluminium combinations with other propellant formulations also confirmed the burning rate augmentation which are almost identical as reported from Dokhan et al. [4] studies. Production of AP in nanosizes of $35-100 \mathrm{~nm}$ was reported by Pivkina et al. [9], and they also described that the nanosized aluminium production comprises $10 \%$ graphite using the mechanical activation method with the size ranging between 20 and $50 \mathrm{~nm}$. The combustion of AP-Al blends with the ratio of $76: 24$ for the pressure ranging between 1 and $6 \mathrm{MPa}$ was tested, which implied that the substitution of micron-sized $(10 \mu \mathrm{m})$ AP using nanosized AP alone could not substantially modify the burning rate and the pressure exponent values as well, whereas the substitution of micron-sized $(97 \mu \mathrm{m}) \mathrm{Al}$ by means of nano- $\mathrm{Al}$ can increase the burning rate almost $>5$ times; however, the values of the pressure exponent retained; then again, concurrent substitution of both AP in micron size and nanosized $\mathrm{Al}$ induced around tenfold of the burning rate increment with respect to their micron-sized equivalent compositions and even reduced the pressure exponent by half which is averaged for the entire tested pressure range. In another study, Popenko et al. [10] stated the burning rate enhancement and reduction in the pressure exponent value of HMXbased condensed systems by including relatively low quantities (1.25-5\%) of ultrafine aluminium particles; however, there is no consequence or undesirable effect towards rheological properties of the modelled propellant due to its addition.

Liu et al. [11] also stated the burning rate increment of propellants because of the addition of nano- $\mathrm{Al}$ as compared with microaluminized counterparts. In contrast, Jayaraman et al. $[12,13]$ reported the significant variations in burning rate pressure exponents $(n)$ of the propellant containing nanoaluminium with respect to its non- and microaluminized counterparts for a wide pressure ranging from 1 to $12 \mathrm{MPa}$, with a close variation of pressure with the step of every $1 \mathrm{MPa}$. With the exception of Dokhan et al. [4] and Jayaraman et al. $[12,13]$, the other investigators on propellant combustion have not reported $n$ to be significantly altered by the addition of ultrafine Al. Indeed, since the aluminium combustion could predominantly be diffusion limited, particularly the propellant burning rate controlled at pressures $(>6 \mathrm{MPa})$, it may be expected to exhibit lower $n$ than the nonaluminized propellant in the maximum tested pressure range up to $10 \mathrm{MPa}$. This was, in fact, shown in tests for wider pressure ranges with close variation in pressure. The variation in pressure exponent " $n$ " of the propellant burning rates containing nanoaluminium is evidently based on fine AP and coarse AP particle sizes and their contents in the formulation. Jayaraman et al. [12] further reported a large family of propellant formulations for comparing those with the plateau and nonplateau burning tendencies, in each case with nonaluminized and aluminized versions, the latter with micro-Al and nano-Al, and with or without burning rate catalysts, and also using two different curing agents, IPDI and TDI.

Iqbal and Liang [14] modelled the viscosity buildup and mechanical properties of the propellants. They found the reduction in viscosity by enhancing the fine AP particle's percentage in the composition, the elongation factor enhances by raising the fine AP particle size, and mechanical strength and elongation significantly decreased with mean AP particle size increment. Jawalkar et al. [15] investigated the viscosity buildup and mechanical properties of the IPDI-/TDI-cured propellants. All these properties for conventional microaluminized propellants have been compared with nanoaluminized counterparts (bicurative system). It is reported that a significant increase in the burning rates and gain in specific impulse can be achieved for nanoaluminized propellants with the bicurative system by comparing with microaluminized propellants, without significant variation in the mechanical properties. In addition, the values obtained for the end of mix viscosity are comparable to the conventional propellant formulations either with TDI or IPDI as the curing agent.

\section{Nanoaluminium Features}

In recent times, the production possibility of nanosized materials has enlarged, and also, the attraction towards generating substances possessing novel properties for developing advanced technologies is commenced. Nanosized materials exhibit substantially favourable qualities, for 
instance, improved catalytic activity, augmentation in reactivity, and reduction in melting temperature. These materials possess distinctive physical and chemical properties when related to their respective micron-sized counterparts and also bulk materials. Actually, the incorporation of metalbased reactive nanomaterials in many applications is accelerating the research and development of nanomaterial production. In general, the nanoparticles are described with the diameter of $<100 \mathrm{~nm}$. They possess unique and excellent properties as compared with those bulk material counterparts. These properties constitute the nanoparticles as suitable materials, particularly to utilize in the applications towards improving the catalytic reactivity of the system. Explicitly, nanosized $\mathrm{Al}(\mathrm{nAl})$ is explored to enhance the performance by improving the burning rate and also the combustion efficiency of the respective energetic systems, directing towards reduction in ignition delays and agglomerate formation, and also burns faster as compared with microaluminized counterparts. Tailoring the propellant burning rate at the specific operating pressure is an essential requirement in any rocket/missile development to fulfil the targeted missions. Most of the times, burning rate modifiers (catalysts) are included in the propellants with very small quantities during the developmental stage of the propellant formulation for tailoring the burning rate.

Nano-Al can be produced from different methods such as inert gas condensation, mechanical attrition, electrical wire exploding technique, and pulsed plasma synthesis process. Amid all these techniques, the electrical wire explosion technique was generally incorporated in the past due to its shape, size, and material purity, and also, these produced quite consistently. A few researchers stated the production and characterization of nanoparticles using the electrical wire explosion technique, using the explosion of a thin $\mathrm{Al}$ wire in the surrounding medium of air [16], and in various inert ambiences such as argon, nitrogen, and helium $[17,18]$. Dependency of particle size distribution due to ambient pressure and other material characterizations of the nano-Al particles generated by single-wire explosion were stated by Sindhu et al. [19]. From these results, it was found that nano-Al particle's shape that is produced through argon and helium ambiences is spherical, whereas it is irregular in nitrogen ambience due to the presence of aluminium nitride. Jayaraman et al. [12] generated the nano-Al particles in nitrogen and argon ambiences by exploding multiple wires in a chamber and characterized them using various techniques.

Typically, such nano-Al powders generated through the wire explosion method are stated to display relatively low melting and ignition temperature, improved specific surface area, and superior reactivity features [7, 20-23]. In general, several investigators have produced the ultrafine $\mathrm{Al}$ powder particle sizes ranging from $100-$ to $200 \mathrm{~nm}$, apart from a few, in which they have utilized the identical process to produce nominal sized particles in few tens of nanometer, i.e., $\$ 50 \mathrm{~nm}[5,13,19]$ over a spread of 5-100 $\mathrm{nm}$. By considering these variations in the range of particle sizes, the "ultrafine" and "superfine" terms are utilized for representing the $\mathrm{Al}$ particles which are usually bigger than few tens of nanometer-sized particles, specifically, in submicrometer ranges. Some other studies have focused on the nano-Al influence towards the thermal decomposition behavior of AP mixtures [11].

The investigation on thermal features using differential thermal analysis (DTA), thermogravimetric analysis (TGA), and differential scanning calorimetry (DSC) of the nanoaluminium particles is carried out and also compared with the samples containing micron-sized aluminium particles by many investigators [20, 21, 23-25]. Apart from these, some researchers have analysed the effect of nano- $\mathrm{Al}$ on thermal decomposition features of propellant/explosive compositions with those comprising ammonium perchlorate (AP) [11] and RDX mixtures [9, 21]. These studies supported that nanoaluminium particles are melting relatively at low temperature level than bulk aluminium; oxidation of nanoaluminium originates even at rather lowtemperature regions, and also, the AP decomposition at low temperature is expedited with the inclusion of nanoaluminium. These results confirm the preservation of extreme burning rates of pressed pellets of dry mixtures containing AP and ultrafine aluminium as stated by Romonadova and Pokhil [3]. Sigman et al. [26] analysed the LPDL (low-pressure deflagration limit) of ultrafine aluminium (Alex-) mixed AP dry-pressed pellets which validated that the LPDL is shifted into a higher pressure range by decreasing the size of $\mathrm{Al}$ particles. Hence, it is postulated that nanoaluminium has relatively more oxide percentage due to more specific surface area, irrespective of the size of oxide skin covered over the surface, and its nature could either be completely crystalline or amorphous; it is a strain process for molten aluminium to crack from the inside across the oxide layers, different for large $\mathrm{Al}$ particles, in which interfaces are vulnerable to crack formation among the crystalline and amorphous islands of the outer oxide skin. DeSena and Kuo [27] investigated the residual energy storage because of high solidification rate during the ultrafine exploded aluminium particles' formation and realised that it is insignificant.

Trunov et al. [25] attempted to rationalise the nanoaluminium oxidation mechanism in the context of the staged oxidation and micron-sized aluminium particle's ignition and related with crystalline phase changes during the oxide formation, as stated from their earlier studies. Rai et al. [28] reported the nanoaluminium particle's oxidation using mass spectrometry, density measurements, and transmission electron microscopy studies. The process of oxygen diffusion through the oxide skin into the core of nanoaluminium particles is focused, that is relatively low proceeding to aluminium melting point and however diffusion process subsequently accelerated.

Kwon et al. [29] displayed dusts of ultrafine aluminium oxidation in air so as to estimate the role of nitrogen in the air for forming aluminium nitride besides the formation of aluminium oxide. Huang et al. [30] carried out the bimodal mixtures of combustion that comprise nano- and micronsized aluminium particle clouds inside the chamber in air and stated the increment in nano-Al/air flame speed as compared with micron-sized aluminum flame speeds. In the 
nano-Al and micro-Al blends, smaller quantities in the former also exhibiting the flame zones are almost distinct as separate regions in which the larger quantities of nano-Al merging as two flame zones apart are formed.

The surface coating influence on nanoaluminium particles and their characterization is stated by few researchers. Jones et al. [31] and Kwok et al. [32] assessed the coated nanoaluminium sample's characterization for various sizes comprising a thick oxide layer, a fluoropolymer, etc., besides the thermal behavior analysis of such type of particle mixing with respect to other few energetic materials, for example, RDX, TNT, and AP. Bocanegra et al. [33] assessed the coating influence on aluminium particles by comparing the burning times of nickel coated and uncoated micron-sized particles and related with respect to its uncoated ultrafine aluminium particles which are supplied by various Russian sources. It was observed that $\mathrm{Ni}$-coated micron-sized aluminium and ultrafine aluminium equally display decrease in burning times; however, the latter one burns relatively faster of the two. Dubois et al. [34] investigated the nanoaluminium and boron particles' coating via grafting polyethylene kind of polymers and also polyurethane on the surface of the metal particles and additionally reported the coated material characterization under various conditions.

\section{Combustion Characteristics of Nanoaluminized Sandwiches and Solid Propellants}

Several investigators examined the influence of ultrafine aluminium in composite solid propellant burning rates, especially those based on AP. As stated in previous studies, Romonodova and Pokhil [3] stated both dry-pressed blends of ultrafine aluminium and AP burning rates. Dokhan et al. [4] examined the propellant mixtures' burning rates containing either unimodal ultrafine particles or micron-sized aluminium samples and also the bimodal particle mixtures including both sizes and tested up to the pressure of 6.9 MPa. It was noticed that the inclusion of ultrafine aluminium augments propellant burning rates via aluminium ignition due to the leading edge flames (LEFs) that are formed above the binder/coarse AP lamina boundary and/or the flames of the binder/fine AP matrix. The report by Ivanov et al. [5] stated that the increment of burning rates due to nanoaluminium addition is around 1.6-2 times with respect to propellant mixtures without containing aluminium for various pressures and levels of aluminium substitution. It was noticed that the propellant burning rates are enhanced almost twice by the ultrafine aluminium addition consistently over the tested pressures, i.e., not much variation in the burning rate pressure exponent for entire tested pressure ranges. They have further examined the bimodal blends of aluminium for other propellant formulations, and outcomes are almost comparable with those of reported ones [4].

3.1. Sandwich Combustion. The influence of nano-Al particles in propellants that burn nearer to the burning surface is clearly distinguished and improves the net heat feedback towards these surfaces $[4,12,13]$. Then again, the LEFs are governing the overall regression rate even for the nanoaluminized ones; hence, the possibility is that nano-Al combustion in the near surface could only support the overall gas phase flame complex and consecutively the LEFs are positioned themselves in the vicinity of burning surface that increases the heat feedback rather than by itself. These results could be affirmed subsequently while investigating the nanoaluminized matrix sandwich burning rates.

Burning rates of sandwiches comprising 15\%,25\%, and $40 \%$ levels of microaluminium or nanoaluminium by mass blended with the binder materials in the middle laminae are examined at three pressures, and the outcomes are displayed in Figures 1(a)-1(c). The leading edge flames (LEFs) are developed at the boundary between the binder layer and AP laminae and control the sandwich burning rates [35]. In particular, the pure binder sandwiches exhibited that the maximum burning rate enhances by increasing the pressure and shifts towards smaller binder lamina thickness. The maximum sandwich burning rate takes place for a specific lamina thickness in which the close interaction between two LEFs is observed.

The sandwiches containing $15 \%$ of nanoaluminium show enhancement in burning rates than those of nonaluminized samples as compared with the samples containing 15\% microaluminium particles. This could be mainly attributable to the easier nanoaluminium particle ignition and from its combustion completion near the burning surface of the sandwiches [4], which ultimately improves the burning rate of sandwiches. However, the sandwich burning rates comprise of $25 \%$ and $40 \%$ micro- or nanoaluminium and almost show similar burning rates as compared with pure-binder sandwiches. These factors clearly emphasize the large-scale nanoaluminium accumulation taking place at the upper levels of solid loading, as not supposed to be favourable to augment the burning rate. These can emphasize that there could be a swapping among the near-surface nanoaluminium ignition, combustion characteristics, and accumulation features for improving the sandwich burning rates. It is noticed that the observation of maximum burning rates of the nanoaluminized sandwiches of larger binder lamina thicknesses as compared to its microaluminized counter parts and it showed relatively higher burning rate than pure binder sandwich samples, as illustrated in Figures 1(a)-1(c). The particulates' addition in the middle binder lamina results in the optimum thickness of the lamina, which exhibits that the maximum burning rate is increased since the particles tend to dilute the binder [36, 37].

Gnanaprakash et al. [38] also proved the earlier burning rate outcome through carrying out the experimental investigation on the combustion of $\mathrm{AP} / \mathrm{HTPB} / \mathrm{Al}$ sandwich propellants. Sandwiches comprise the HTPB matrix, mixed of $\sim 45 \mathrm{~nm}$ Al with the fine AP particles with the sizes of either 5 or $45 \mu \mathrm{m}$, fabricated over three various middle lamina thickness ranges: thin (100 to $170 \mu \mathrm{m}$ ), intermediate (200 to $280 \mu \mathrm{m}$ ), and finally thick (370 to $480 \mu \mathrm{m}$ ). They performed the tests for the pressures ranging from 1 to $12 \mathrm{MPa}$; however, the emphasis was given over the midpressure ranges from 2 to $6 \mathrm{MPa}$. It was noticed at high-pressure burning of about 
$>6 \mathrm{MPa}$ that additional influence of LEF anchored above large-sized fine AP particles (PLEF - particle leading edge flame) and the significance of the LEF number density above the burning surface (distance comparison among the adjacent coarse AP particles and the LEF distancing from the burning surface). Hence, abnormal tendency of the specific nano-Al propellant compositions does not exhibit any burning rate increment, and also, the influence of the addition of AP particles and their size distribution is described.

3.2. Propellant Combustion. The systematic work by Jayaraman et al. $[12,13,39-41]$ reported different varieties of bimodal sizes of AP/HTPB composite propellant's burning rates for the pressure ranging from 1 to $12 \mathrm{MPa}$, utilising both curing agents such as IPDI (considerable binder melt flow effects) and TDI (observation of a smaller amount of binder melt flow effects) of the HTPBbased binder scheme. And also, validating the preceding typical results of the increment factor from 2 or 3 over its steady-state burning rates from the usual various working environments, these results were accomplished to recognise the typical series of the operational states through slight or without improvement in stable burning rates (plateau burning rate trends) or atypical regime, for example, midpressure or high-pressure extinction. Based on the propellant compositions, for instance, while the maximum-sized fine AP particle systems are executed over different fine AP contents with the smallest fine AP size of either $5 \mu \mathrm{m}$ or both, the coarse AP size is increased up to $450 \mu \mathrm{m}$ with the HTPB as the fuel and curing agents as TDI or IPDI (implicitly emphasize the little effect due to $\mathrm{nAl}[12]$ ); it was noticed that the steady-state burning rate enhancement is much more or even less than a factor of 2 .

Figure 2 shows that the plateau effects are almost nonexistent for non- and microaluminized propellants containing $53 \mu \mathrm{m}$ and $75 \mu \mathrm{m}$ fine AP particles over the tested pressure ranges, whereas the corresponding $5 \mu \mathrm{m}$ fine AP propellants exhibit such effects, except for a slight low-exponent behavior of the nonaluminized $53 \mu \mathrm{m}$ fine AP formulation at the pressure of around 6-8 MPa. When the size of fine AP is decreased from $75 \mu \mathrm{m}$ to $5 \mu \mathrm{m}$ in the nanoaluminized formulations, the burning rate is slightly increased at low pressures, whereas at elevated pressure conditions, the fine AP size effect is minimal. The propellant formulations lacking nanoaluminium exhibit that the pressure exponents are comparatively small for $5 \mu \mathrm{m}$ fine AP particles than the $75 \mu \mathrm{m}$ and $53 \mu \mathrm{m}$ fine AP, as expected, because of its more binder melt flow effect. Also, note that the usual trend of higher fine AP size ensuing in lower burning rates is restored between the 53 and $75 \mu \mathrm{m}$ fine AP propellants regardless of the presence of either type of aluminium tested. Figure 3 displays the influence of the propellants comprising more than that of $10 \%$ of nanoaluminium, and it is observed that there is hardly any burning rate enhancement. This imparts that the net heat feedback from the surface proximity nanoaluminium combustion towards the burning surface almost reached to a certain form of saturation. It is noticed that $15 \%$ microaluminized propellants exhibit low burning rate, as contrasted with $10 \%$ and $18 \%$ microaluminium-included propellants, which indicates the competition features among the heat sink effect and the influence of locally accelerated regressions.

Propellant burning rates are reduced of about $10-20 \%$ as the nanoaluminium size is raised from $55 \mathrm{~nm}$ to $70 \mathrm{~nm}$ as represented in Figure 4. This could be due to the reduction in surface area-to-volume ratio of aluminium which subsides the particle's reactivity. In contrast, the burning rate is enhanced with aluminium particle size in case of microaluminized ones. The aluminium size increment resulting towards reduction in the mobility of particles, number density, and the accumulation tendency thus enhances the propellant burning rates. The nanoaluminium burning proximity to the surface and also from further heat transfers via conduction and radiation towards the propellant increases the burning rates. The burning rate trends of the plateau and mesa displayed of the respective nonaluminized and microaluminized propellants are almost eliminated by comparing with nanoaluminized propellants. As a conflict, the inclusion of microaluminium in the propellant marginally reduces the burning rate. The plateau and the mesa burning rate trends exhibiting in the nonaluminized propellant compositions are distorted, however not completely eradicated by comparing with aluminized propellants. This could be due to the replacement of the coarse AP particles for including microaluminium for maintaining the total solid loading level. In general, the burning rates are mainly governed through the heat transfer process from the aluminium particles' ignition nearer to the burning surface and proximity-complete combustion of nanoaluminium particles above the burning surface of the propellant. Combustion of nanoaluminium is diffusion limited at high pressure ranges, causing with low-pressure exponents in burning rates of the nanoaluminized propellant over these pressure ranges.

The three tested propellant compositions of blended nano- and microaluminium for the ratios of $80 / 20,50 / 50$, and $20 / 80$, along with the inclusion of $100 \%$ nano- and microaluminium which is investigated hitherto, are examined in the matrixes and propellants with a total aluminium percentage of 15 in the composition that is considered in these studies. Figure 5 represents the burning rates of the bimodal mixture of aluminized propellants which display identical burning rate trends as stated in earlier reports $[25,29]$. The burning rate reduction of the propellant that is filled with $100 \%$ microaluminium with respect to the nonaluminized ones is counterbalanced through partial substitution of an even $20 \%$ addition of microaluminium by nanoaluminium at higher pressure ranges. This demonstrates the binder melt flow influence over the burning rate is foremost over the intermediate pressures which are mainly subdued through the heat feedback through the surface proximity of nanoaluminium particle combustion at elevated pressure ranges. Additional increment of the nanoaluminium content increases the burning rate of the propellant than the nonaluminized compositions for the 


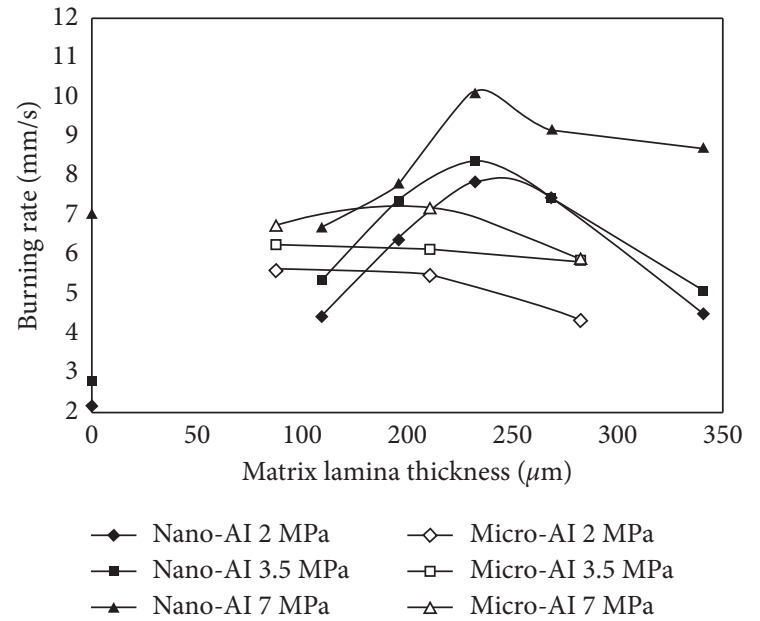

(a)

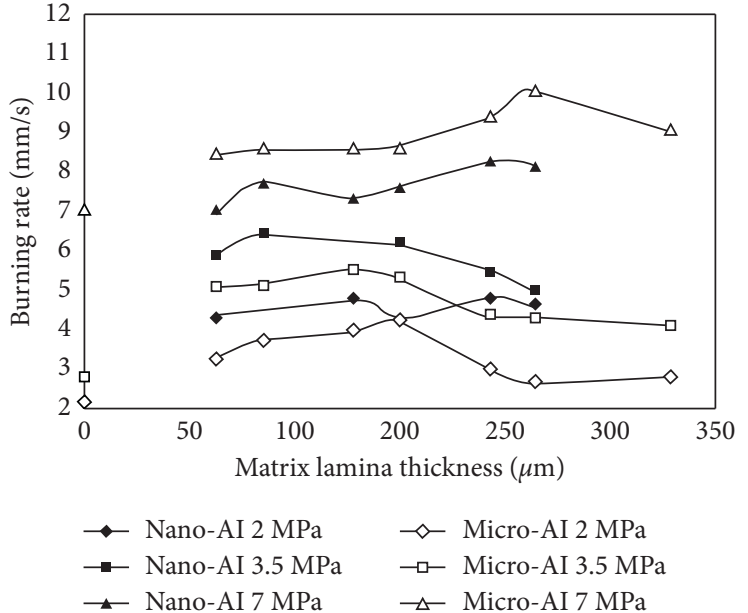

(b)

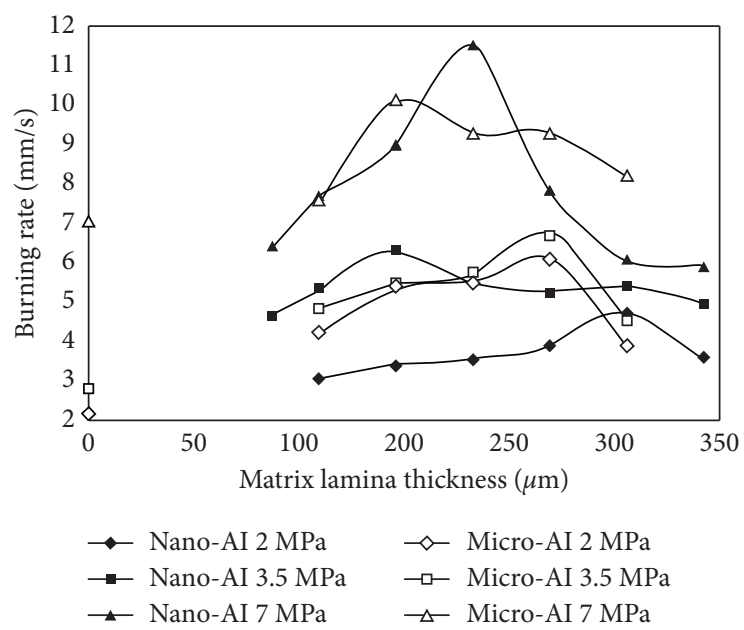

(c)

FigURE 1: Effect of binder lamina thickness of the sandwich burning rates containing various aluminium percentages in the binder composition. (a) Addition of $15 \%$ micro- $\mathrm{Al}$ and nano- $\mathrm{Al}$ in the binder laminae. (b) Addition of $25 \%$ micro- $\mathrm{Al}$ and nano- $\mathrm{Al}$ in the binder laminae. (c) Addition of $40 \%$ micro- $\mathrm{Al}$ and nano- $\mathrm{Al}$ in the binder laminae.

total tested pressure ranges; however, this could be saturated at the nanoaluminium level of $80 \%$ at the bimodal blend. Moreover, the plateau burning rate trends are flushed out as microaluminium is substituted by more than $20 \%$ of nanoaluminium, albeit midpressure extinction happens at the respective bimodal aluminized matrix blends. These factors substantiate that the surface proximity combustion increases the net heat feedback from the nanoaluminium combustion towards the propellant regression surface which mainly controls the propellant burning rates, prevailing as compared to other mechanisms from the LEFs formed above the coarse AP particles.

\section{Estimation of Aluminium Agglomerates from the Combustion of Nanoaluminized Sandwiches and Propellants}

It is observed that the mean sizes of condensed phase combustion products (CCPs) at $0.1 \mathrm{MPa}$ are around
$6.6 \mu \mathrm{m}$ of the propellant comprising nanoaluminium with the size of $150 \mathrm{~nm}$, as compared with the size of $13 \mu \mathrm{m}$ CCPs from the $30 \mu \mathrm{m}$-sized parent aluminium comprised propellants, as reported by Galfetti et al. [8]. In another study, Dokhan et al. [4] reported the size estimation of "non-smoke combustion residue (NSCR)" from bimodal mixtures of micrometre-sized and ultrafine aluminized propellants. Jayaraman et al. [41] quenched the binder and fine AP matrixes at high pressure ranges to examine the surfaces and observed that the nanoaluminium particles are comparatively accumulated in a large scale with the size of around $50 \mathrm{~nm}$, becoming into clusters of around 4-5 $\mu \mathrm{m}$ size ranges. Figure 6 shows aluminium agglomerates recovered at the burning surface from four types of propellants using the quench collection apparatus at the pressure of $6 \mathrm{MPa}$. The huge clusters of aluminium particles exhibited over the size ranging from $\sim 1$ to $5 \mu \mathrm{m}$; however, this is again within the size ranges of smoke oxide particles. 


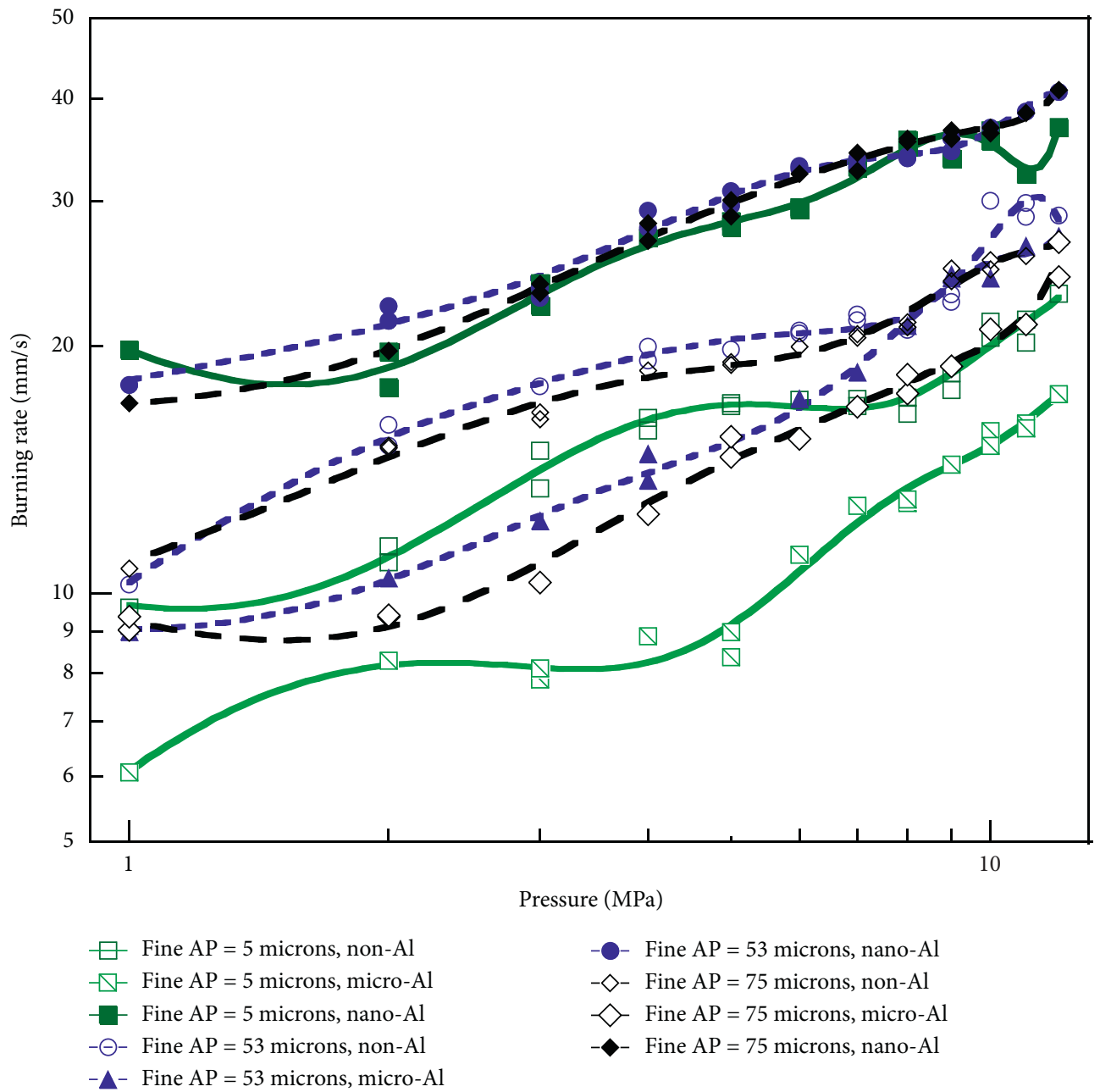

Figure 2: Propellant burning rates containing fine AP $(5 \mu \mathrm{m}, 53 \mu \mathrm{m}$, and $75 \mu \mathrm{m}) / \mathrm{binder}=60 / 40$, coarse AP size $=450 \mu \mathrm{m}$, and TDI cured.

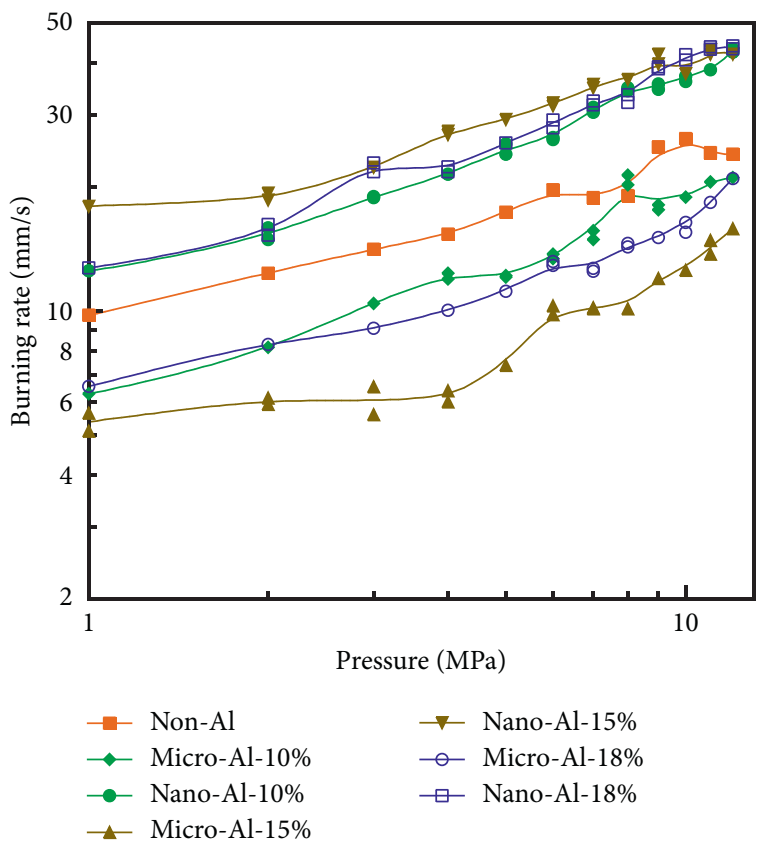

Figure 3: Burning rates of propellants and matrixes for different aluminium levels with the fine AP $(5 \mu \mathrm{m}) / \mathrm{binder}=65 / 35$, coarse AP size $=450 \mu \mathrm{m}$, and IPDI cured. 


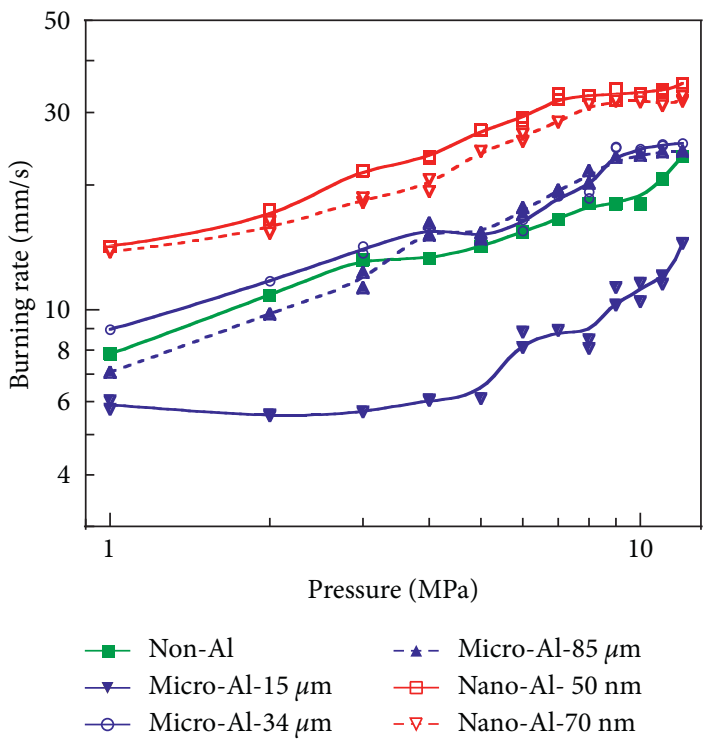

FIGURE 4: Propellant burning rates containing different aluminium sizes, fine AP $(20 \mu \mathrm{m}) / \mathrm{binder}=60 / 40$, coarse AP size $=450 \mu \mathrm{m}$, and IPDI cured.

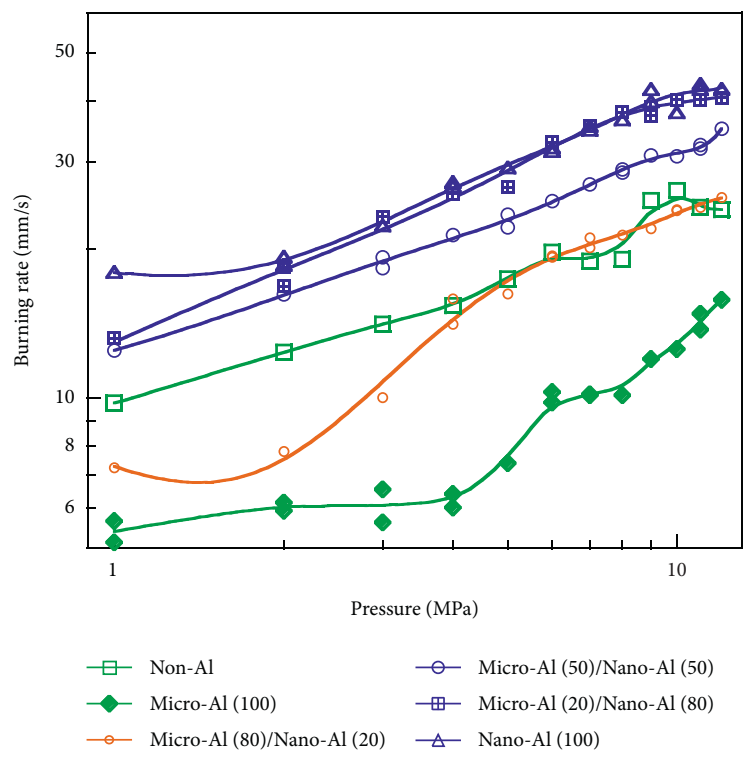

FIGURE 5: Burning rates of the non- and bimodal aluminized propellants.

From Table 1, it can be seen that the agglomeration factor presented by Tejasvi et al. [43] is in the range from 24 to 46 , and from 40 to 50 . By considering the work of Jayaraman et al. [41, 42], the wider agglomeration factor range is mainly caused from smaller-sized nanoaluminium and its broader operating pressure ranges. In contrast, agglomeration factor is within the limited deviation range owing to short pressure range and relatively high due to low pressures as well [8]. In these observations, aluminium particles with the size of $450 \mathrm{~nm}$ are utilized in which the agglomeration factor was restricted within the range of
24-46 even though the tests are performed for wide pressure ranges. However, the sizes of the microaluminium agglomerates are around 28 to $136 \mu \mathrm{m}$, with the agglomeration factor from 1.5 to 8 [44].

\section{Mechanical Properties}

The propellants are mixed in a mini-mixer with the maximum capacity of 500 grams for mechanical testing. Universal Testing Machine (Instron, Germany) with the maximum load capacity of $30 \mathrm{kN}$ has been used for the present study. The 


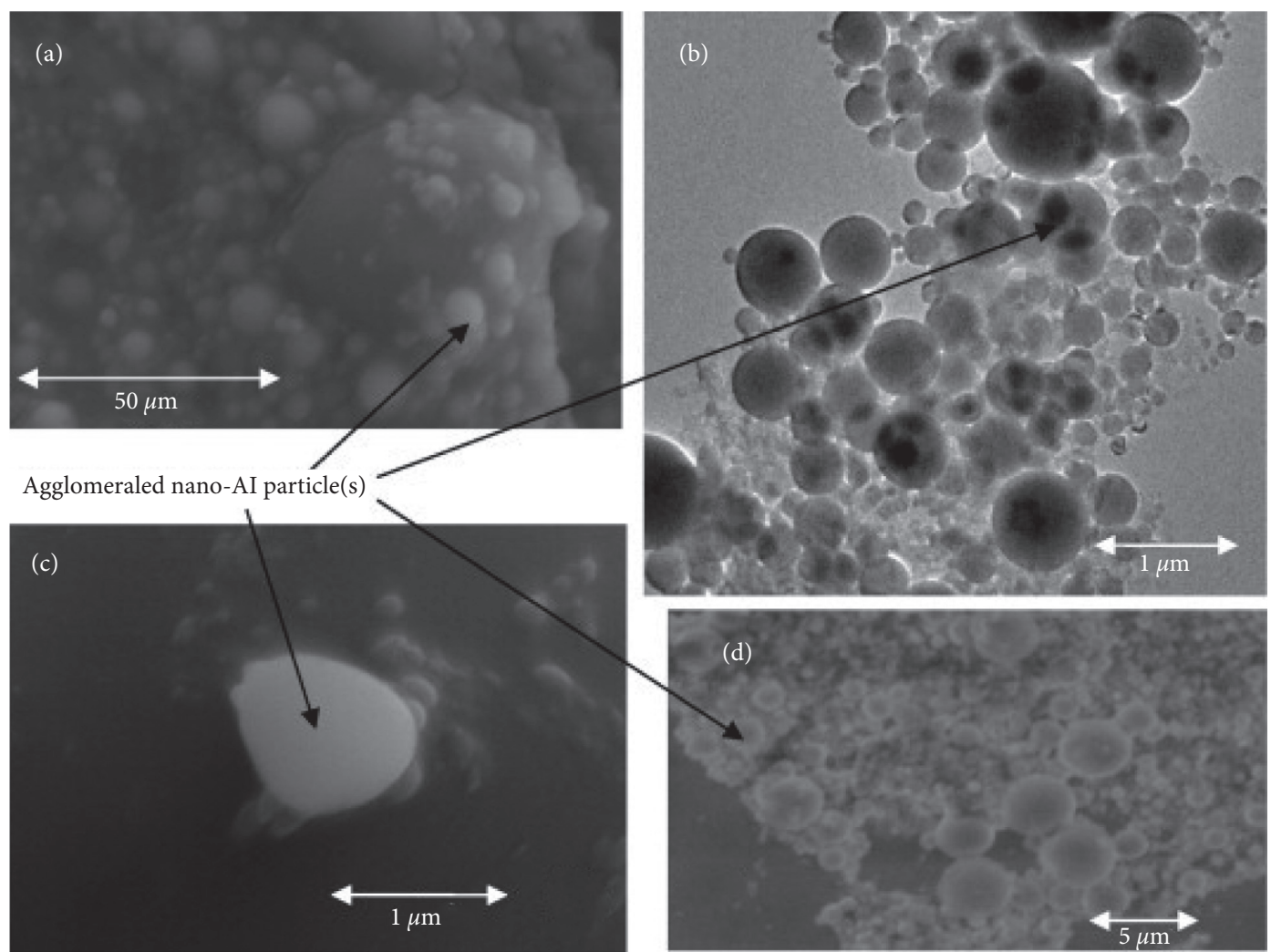

FIGURE 6: SEM/TEM micrographic images of nanoaluminized samples quench collected at $4 \mathrm{MPa}$ from the propellants of various coarse and fine AP sizes: (a) 250 and $75 \mu \mathrm{m}$; (b) 350 and $75 \mu \mathrm{m}$; (c) 450 and $53 \mu \mathrm{m}$; (d) 450 and $75 \mu \mathrm{m}$.

TABLE 1: Agglomerate size studies for nanoaluminized propellants containing AP.

\begin{tabular}{|c|c|c|c|c|c|}
\hline \multirow[b]{2}{*}{ Ref. } & \multirow[b]{2}{*}{ Propellant } & \multicolumn{4}{|c|}{ Experimental } \\
\hline & & Pressure $(\mathrm{MPa})$ & Facility & $\operatorname{Dag}(\mu \mathrm{m})$ & $\begin{array}{c}\text { Agg. fact. }=\text { Agg. } \\
\text { size/parent Al } \\
\text { size }\end{array}$ \\
\hline$[41,42]$ & $\begin{array}{c}\text { AP: } 71 \% \text {, NAl: 15\%, NAl size: } 50 \mathrm{~nm} \text {, HTPB: } \\
12.5 \%\end{array}$ & $4,6,8$ & $\begin{array}{c}\text { QPCB (quench particle collection } \\
\text { bomb) }\end{array}$ & $1-5$ & $20-100$ \\
\hline [8] & $\begin{array}{c}\text { AP: } 65.3 \% \text {, NAl: 15\%, NAl: } 300 \text { nm, HTPB: } \\
17 \%\end{array}$ & $2,2.5$ & Combustion photograph & 13 & $40-50$ \\
\hline [43] & $\begin{array}{c}\text { AP: } 68 \% \text {, UFAL: } 18 \% \text {, UFAL size: } \sim 450 \mathrm{~nm} \text {, } \\
\text { HTPB: } 12.5 \%\end{array}$ & $2,4,6,8$ & QPCB & $11-21$ & $24-46$ \\
\hline
\end{tabular}

cured propellant slab with the thickness of $\sim 5 \mathrm{~mm}$ has been cut into a tumble shape using the die holder. The crosshead speed of the spindle is $50 \mathrm{~mm} /$ minute. This experiment has been carried out at room temperature. The propellant piece has been subjected to load until it breaks. Figure 7 shows the percentage of elongation, initial modulus, and modulus of elasticity of the cured non-, micro-, and nanoaluminized propellants. The percentage of elongation increases with the fine AP particle size, whereas the addition of aluminium particles reduces the elongation strength. Higher-size fine AP particles have lesser number density than small-size particles. It leads to reduction in elongation strength, noted in the past work $[14,15]$. Inclusion of aluminium particles is done with the replacement coarse AP particles, and also, the higher number density of the particles reduces the percentage of elongation. Initial modulus of the nonaluminized propellant is increased, when $75 \mu \mathrm{m}$ is replaced by $105 \mu \mathrm{m}$, and is reduced in aluminized propellants. Higher number density of the particles in the propellant mixture reduces the initial modulus. Modulus of elasticity of the aluminized propellants 


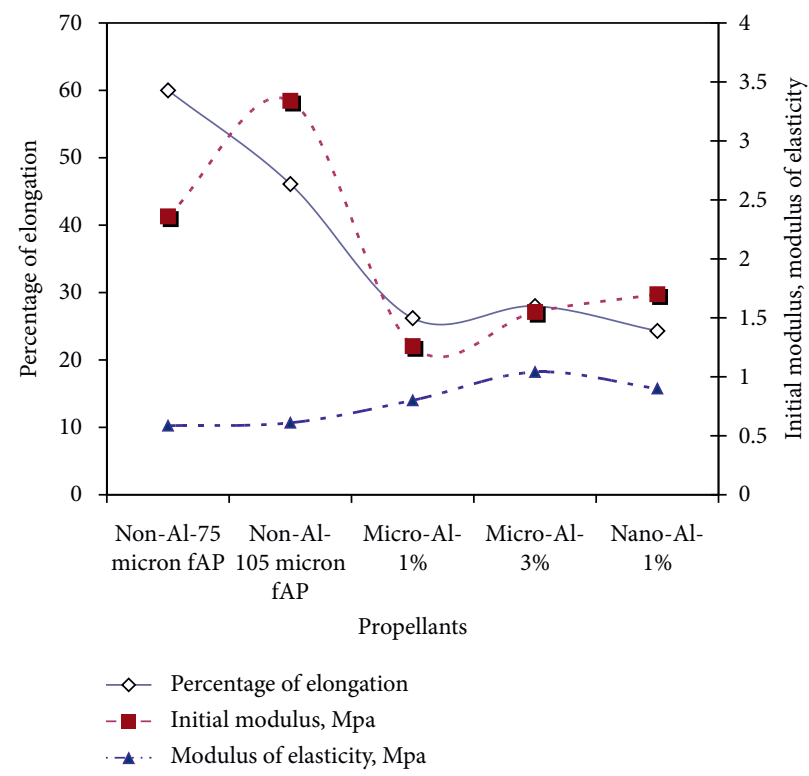

Figure 7: Mechanical properties of the propellants.

shows greater value than their nonaluminized counterpart. This may be due to the adhesive force between the polymer and aluminium particles.

\section{Conclusion}

In the combustion of solid propellants, several reports have shown the influence of "ultrafine" aluminium particles with the size of $\sim 100 \mathrm{~nm}$ and nanoparticles with the size of about less than $100 \mathrm{~nm}$. A single nanoparticle comprises only few hundreds of atoms; hence, the surface energy of this type of particles is rather distinctive as compared with bulk materials.

In the sandwich combustion, the burning rate is enhanced by $\sim 20-30 \%$ for about $15 \%$ solid loading in the binder lamina with nanoaluminium, with subsequent addition of nanoaluminium exhibiting results of almost comparable burning rates with respect to its nonaluminized counterpart. The burning surface profiles of the sandwiches suggest that nanoaluminium combusted near the burning surface which contributes as an important heat feedback mechanism for influencing the burning rate.

The inclusion of nanoaluminium results in an enormous increase $(\sim 100 \%)$ in propellant burning rates. For smaller fine $\mathrm{AP}$ and coarse AP particles, the burning rate enhancement of the nanoaluminized propellant over its nonaluminized compositions is marginal. Appreciable changes occur in the burning rate pressure exponent between the nanoaluminized and nonaluminized propellants over the tested pressure ranges from 1 to $12 \mathrm{MPa}$. As microaluminium is substituted with more than $20 \%$ by nanoaluminium, the plateau burning trends are almost flushed away albeit midpressure extinction takes place in the respective bimodal aluminized matrixes. In general, nanoaluminium combustion almost completely occurred at the proximity of the burning surface that increases the net heat feedback which is considered as a rate-controlling mechanism for the propellant formulations containing nanoaluminium.

Size of the nanoaluminium accumulation cluster is $\sim 4 \mu \mathrm{m}$ on the propellant burning surface; however, the microaluminium clusters are estimated as relatively large in size. Similarly, self-quenched matrix mixtures comprise of fine AP and binder, and nanoaluminium displays substantial accumulation of aluminium particles with the sizes of $\sim 4-5$ $\mu \mathrm{m}$ clusters; however, microaluminium accumulation comparatively formed into large-sized clusters. The reduction in aluminium agglomerate size is observed by increasing the chamber pressure and also the quenching distances away from burning surfaces. At elevated pressure conditions, the LEFs approach nearer to the burning surface and also efficiently ignite the aluminium agglomerates and decrease their sizes. The prospect of aluminium agglomeration and its size is comparatively low at elevated pressure level and with larger initial aluminium sizes. This factor is conducive that approaches towards reduced propellant smoke applications for diminishing the exhaust signature point of view, without considerably forfeiting propellant energetics. The two-phase flow losses to thrust and the feasibility of slag accumulation are appreciably prevailed over with smaller-sized aluminium agglomerates and consequently their combustion efficiency using UFAL (ultrafine aluminium) in propellants, as compared with micron-sized aluminized propellants which are formed in large-sized $\mathrm{Al}$ agglomerates. Percentage of elongation and initial modulus are decreased when the coarse AP particles are replaced by aluminium. Modulus of elasticity improves with the addition of aluminium particles in the propellant.

\section{Conflicts of Interest}

The authors declare that they have no conflicts of interest. 


\section{Authors' Contributions}

Kandasamy Jayaraman and Ponnurengam Malliappan Sivakumar contributed equally to this work.

\section{References}

[1] M. Summerfield, G. S. Sutherland, M. J. Webb, H. J. Tabak, and K. Hall, "Burning mechanisms of ammonium perchlorate propellants," in Solid Propellant Rocket Research, M. Summerfield, Ed., vol. 1, Academic Press, Progress in Astronautics and Aeronautics, pp. 141-182, 1960.

[2] M. W. Beckstead, R. L. Derr, and C. F. Price, "A model of composite solid-propellant combustion based on multiple flames," AIAA Journal, vol. 8, no. 12, pp. 2200-2207, 1970.

[3] L. D. Romonadova and P. K. Pokhil, "Action of silica on the burning rates of ammonium perchlorate compositions," Combustion, Explosion and Shock Waves, vol. 6, pp. 258-261, 1970.

[4] A. Dokhan, E. W. Price, J. M. Seitzman, and R. K. Sigman, "The effects of bimodal aluminum with ultrafine aluminum on the burning rates of solid propellants," Proceedings of the Combustion Institute, vol. 29, no. 2, pp. 2939-2946, 2002.

[5] Y. F. Ivanov, M. N. Osmonoliev, V. S. Sedoi et al., "Productions of ultra-fine powders and their use in high energetic compositions," Propellants, Explosives, Pyrotechnics, vol. 28, no. 6, pp. 319-333, 2003.

[6] L. T. De Luca, L. Galfetti, F. Severini et al., "Burning of nanoaluminized composite rocket propellants," Combustion, Explosion and Shock Waves, vol. 41, no. 6, pp. 680-692, 2005.

[7] L. Galfetti, L. T. D. Luca, F. Severini et al., "Nanoparticles for solid rocket propulsion," Journal of Physics: Condensed Matter, vol. 18, no. 33, pp. S1991-S2005, 2006.

[8] L. Galfetti, L. T. DeLuca, F. Severini, G. Colombo, L. Meda, and G. Marra, "Pre and post-burning analysis of nano-aluminized solid rocket propellants," Aerospace Science and Technology, vol. 11, no. 1, pp. 26-32, 2007.

[9] A. N. Pivkina, Y. V. Frolov, and D. A. Ivanov, "Nanosized components of energetic systems: structure, thermal behavior, and combustion," Combustion, Explosion, and Shock Waves, vol. 43, no. 1, pp. 51-55, 2007.

[10] E. M. Popenko, A. A. Gromov, Y. Y. Shamina, A. P. Il'in, A. V. Sergienko, and N. I. Popok, "Effect of the addition of ultrafine aluminum powders on the rheological properties and burning rate of energetic condensed systems," Combustion, Explosion, and Shock Waves, vol. 43, no. 1, pp. 46-50, 2007.

[11] L. Liu, F. Li, L. Tan, L. Ming, and Y. Yi, "Effects of nanometer $\mathrm{Ni}, \mathrm{Cu}, \mathrm{Al}$ and $\mathrm{NiCu}$ powders on the thermal decomposition of ammonium perchlorate," Propellants, Explosives, Pyrotechnics, vol. 29, no. 1, pp. 34-38, 2004.

[12] K. Jayaraman, K. V. Anand, S. R. Chakravarthy, and R. Sarathi, "Effect of nano-aluminium in plateau-burning and catalyzed composite solid propellant combustion," Combustion and Flame, vol. 156, no. 8, pp. 1662-1673, 2009.

[13] K. Jayaraman, K. V. Anand, D. S. Bhatt, S. R. Chakravarthy, and R. Sarathi, "Production, characterization, and combustion of nanoaluminum in composite solid propellants," Journal of Propulsion and Power, vol. 25, no. 2, pp. 471-481, 2009.

[14] M. M. Iqbal and W. Liang, "Modeling of composite propellant properties based on polymer rheology," in Proceedings of the 42nd AIAA/ASME/SAE/ASEE Joint Propulsion Conference \&
Exhibit, AIAA 2006, pp. 9-12, Sacramento, CA, USA, July 2006.

[15] S. N. Jawalkar, D. Mehilal, R. Kurva, P. P. Singh, and B. Bhattacharya, "Influence of bicurative on processibility of composite propellant," Defence Science Journal, vol. 57, no. 5, pp. 669-675, 2007.

[16] V. Sabari Giri, R. Sarathi, S. R. Chakravarthy, and C. Venkataseshaiah, "Studies on production and characterization of nano- $\mathrm{Al}_{2} \mathrm{O}_{3}$ powder using wire explosion technique," Materials Letters, vol. 58, no. 6, pp. 1047-1050, 2004.

[17] T. K. Sindhu, S. R. Chakravarthi, R. Jayaganthan, and R. Sarathi, "Studies on generation and characterization of nano aluminium nitride using wire explosion technique," Synthesis and Reactivity in Inorganic, Metal-Organic, and Nano-Metal Chemistry, vol. 36, no. 1, pp. 53-58, 2006.

[18] R. Sarathi, T. K. Sindhu, and S. R. Chakravarthy, "Generation of nano aluminium powder through wire explosion process and its characterization," Materials Characterization, vol. 58, no. 2, pp. 148-155, 2007.

[19] T. K. Sindhu, R. Sarathi, and S. R. Chakravarthy, "Generation and characterization of nano aluminium powder obtained through wire explosion process," Bulletin of Materials Science, vol. 30, no. 2, pp. 187-195, 2007.

[20] Y.-S. Kwon, J.-S. Moon, A. P. Ilyin, A. A. Gromov, and E. M. Popenko, "Estimation of the reactivity of aluminum superfine powders for energetic applications," Combustion Science and Technology, vol. 176, no. 2, pp. 277-288, 2004.

[21] Q. S. M. Kwok, R. C. Fouchard, A.-M. Turcotte, P. D. Lightfoot, R. Bowes, and D. E. G. Jones, "Characterization of aluminum nanopowder compositions," Propellants, Explosives, Pyrotechnics, vol. 27, no. 4, pp. 229-240, 2002.

[22] G. H. Lee, J. H. Park, C. K. Rhee, and W. W. Kim, "Fabrication of Al nano- powders by pulsed wire evaporation (PWE) method," Journal of Industrial and Engineering Chemistry, vol. 9, no. 1, pp. 71-75, 2003.

[23] M. M. Mench, K. K. Kuo, C. L. Yeh, and Y. C. Lu, "Comparison of thermal behavior of regular and ultra-fine aluminum powders (alex) made from plasma explosion process," Combustion Science and Technology, vol. 135, no. 1-6, pp. 269-292, 1998.

[24] C. E. Johnson, S. Fallis, A. P. Chafin et al., "Characterization of nanometer- to micron-sized aluminum powders: size distribution from thermogravimetric analysis," Journal of Propulsion and Power, vol. 23, no. 4, pp. 669-682, 2007.

[25] M. A. Trunov, S. M. Umbrajkar, M. Schoenitz, J. T. Mang, and E. L. Dreizin, "Oxidation and melting of aluminum nanopowders," The Journal of Physical Chemistry B, vol. 110, no. 26, pp. 13094-13099, 2006.

[26] R. K. Sigman, E. K. Zachary, S. R. Chakravarthy, J. M. Freeman, and E. W. Price, "Preliminary Characterization of the Combustion Behavior of Alex in Solid Propellants," in Proceedings of the 34th JANNAF Combustion Meeting, West Palm beach, FL, USA, 1997.

[27] J. T. DeSena and K. K. Kuo, "Evaluation of stored energy in ultrafine aluminum powder produced by plasma explosion," Journal of Propulsion and Power, vol. 15, no. 6, pp. 794-800, 1999.

[28] A. Rai, K. Park, L. Zhou, and M. R. Zachariah, "Understanding the mechanism of aluminium nanoparticle oxidation," Combustion Theory and Modelling, vol. 10, no. 5, pp. 843-859, 2006.

[29] Y.-S. Kwon, A. A. Gromov, A. P. Ilyin, E. M. Popenko, and G.-H. Rim, "The mechanism of combustion of superfine 
aluminum powders," Combustion and Flame, vol. 133, no. 4, pp. 385-391, 2003.

[30] Y. Huang, G. A. Risha, V. Yang, and R. A. Yetter, "Combustion of bimodal nano/micron-sized aluminum particle dust in air," Proceedings of the Combustion Institute, vol. 31, no. 2, pp. 2001-2009, 2007.

[31] D. E. G. Jones, R. Turcotte, R. C. Fouchard, Q. S. M. Kwok, A.-M. Turcotte, and Z. Abdel-Qader, "Hazard characterization of aluminum nanopowder compositions," Propellants, Explosives, Pyrotechnics, vol. 28, no. 3, pp. 120-131, 2003.

[32] Q. S. M. Kwok, C. Badeen, K. Armstrong, R. Turcotte, D. E. G. Jones, and V. Y. Gertsman, "Hazard characterization of uncoated and coated aluminium nanopowder compositions," Journal of Propulsion and Power, vol. 23, no. 4, pp. 659-668, 2007.

[33] P. E. Bocanegra, C. Chauveau, and I. Gokalp, "Experimental studies on the burning of coated and uncoated micro- and nano sized aluminium particles," Aerosp Sci Technol, vol. 11, no. 1, pp. 33-38, 2007.

[34] C. Dubois, P. G. Lafleur, C. Roy, P. Brousseau, and R. A. Stowe, "Polymer-grafted metal nanoparticles for fuel applications," Journal of Propulsion and Power, vol. 23, no. 4, pp. 651-658, 2007.

[35] E. W. Price, J. K. Sambamurthi, R. K. Sigman, and R. R. Panyam, "Combustion of ammonium perchloratepolymer sandwiches," Combustion and Flame, vol. 63, no. 3, pp. 381-413, 1986.

[36] S. T. Lee, Multidimensional Effects in Composite Propellant Combustion, Ph. D Thesis, Georgia Institute of Technology, Atlanta, GA, USA, 1991.

[37] M. Navaneethan, V. Srinivas, and S. R. Chakravarthy, "Coupling of leading edge flames in the combustion zone of composite solid propellants," Combustion and Flame, vol. 153, no. 4, pp. 574-592, 2008.

[38] K. Gnanaprakash, S. R. Chakravarthy, and R. Sarathi, "Combustion mechanism of composite solid propellant sandwiches containing nano-aluminium," Combustion and Flame, vol. 182, pp. 64-75, 2017.

[39] K. Jayaraman, K. V. Anand, S. R. Chakravarthy, and R. Sarathi, "Production and characterization of nano aluminum and its effect in solid propellant combustion," in Proceedings of the 45th AIAA Aerospace Science Meeting and Exhibit, Reno, NV, USA, January 2007.

[40] K. Jayaraman, S. R. Chakravarthy, and R. Sarathi, "Behavior of nano-aluminum in solid propellant combustion," in Proceedings of the 44th AIAA/ASME/SAE/ASEE Joint Propulsion Conference, Hartford, CT, USA, July 2008.

[41] K. Jayaraman, S. R. Chakravarthy, and R. Sarathi, "Quench collection of nano-aluminium agglomerates from combustion of sandwiches and propellants," Proceedings of the Combustion Institute, vol. 33, no. 2, pp. 1941-1947, 2011.

[42] K. Jayaraman, S. R. Chakravarthy, and R. Sarathi, "Accumulation of nano-aluminium during combustion of composite solid propellant mixtures," Combustion, Explosion, and Shock Waves, vol. 46, no. 1, pp. 21-29, 2010.

[43] K. Tejasvi, V. Venkateshwara Rao, Y. Pydi Setty, and K. Jayaraman, "Ultra-fine aluminium characterization and its agglomeration features in solid propellant combustion for various quenched distance and pressure," Propellants, Explosives, Pyrotechnics, vol. 45, no. 5, pp. 714-723, 2020.

[44] K. Tejasvi, V. Venkateshwara Rao, Y. PydiSetty, and K. Jayaraman, "Studies on aluminum agglomeration and combustion in catalyzed composite propellants," Combustion, Explosion, and Shock Waves, vol. 57, no. 2, pp. 203-214, 2021. 